\title{
Experimental study of the D-OSKIL mechanism for controlling the stick-slip oscillations in a drilling laboratory testbed
}

\author{
Haochuan Lu \\ Electrical\&Electronic Engineering \\ Nanyang Technological University, Singapore \\ haochuanlu@gmail.com
}

\author{
Jonathan Dumon \\ CNRS, Gipsa-Lab \\ Grenoble, France
Carlos Canudas-de-Wit
CNRS, Gipsa-Lab
Grenoble, France \\ Jonathan.Dumon@gipsa-lab.inpg.fr carlos.canudas-de-wit@gipsa-lab.inpg.fr
}

\begin{abstract}
The presence of harmful stick-slip oscillations in oil well drillstrings has attracted the attention of the control community in recent years. The control law named D-OSKIL mechanism which uses the weight on the bit (WoB) force as an additional control variable to extinguish limit cycles has been investigated [1]. This paper reports experimental implementation of such a mechanism, in a laboratory testbed. We also provide details of the experimental process and the obtained drilling performance. Results show that the stick-slip oscillations can be effectively suppressed by this mechanism.
\end{abstract}

\section{INTRODUCTION}

Deep wells for exploration of oil are drilled with a rotary drillstring system. Different drillstring oscillations are an important cause for premature failure of drillstring components and drilling inefficiency. The occurrence of stick-slip oscillation as a common and damaging phenomena given at the bottom-hole assembly (BHA), i.e., the top of the drillstring rotates with constant rotary speed whereas the bit (cutting tool) rotary speed varies between zero and up to six times the rotary speed measured at the surface, has attracted attention of control community in the last decade.

There are several feasible alternatives to suppress stickslip oscillations. This project adopted the mechanism named drilling oscillation killer (D-OSKIL) that is proposed in the paper [1]. We implemented it on an experimental setup that emulates a real oil drilling platform. The mechanism mainly utilizes the weight on the bit (WoB) force as an additional control variable to eliminate stick-slip oscillations. An important property of D-OSKIL is that it allows recovering the nominal operation condition (the WoB recovers its nominal drilling value) while oscillations are suppressed.

The next section describes the testbed assembled in this project. Then we reviews the system model and the DOSKIL mechanism. Subsequent section are devoted to the experimental validation of the D-OSKIL mechanism, and the last part gives the conclusion.

\section{TESTBED DESCRIPTION}

An experimental setup which emulates the oil drilling system in the field was constructed to investigate the performance of D-OSKIL mechanism on a real platform. It can reproduces similar stick-slip oscillations as the ones reported in field tests. The experimental setup as illustrated in Fig.1 and in Fig.2 consists of following parts:

ROTARY SYSTEM is composed of:

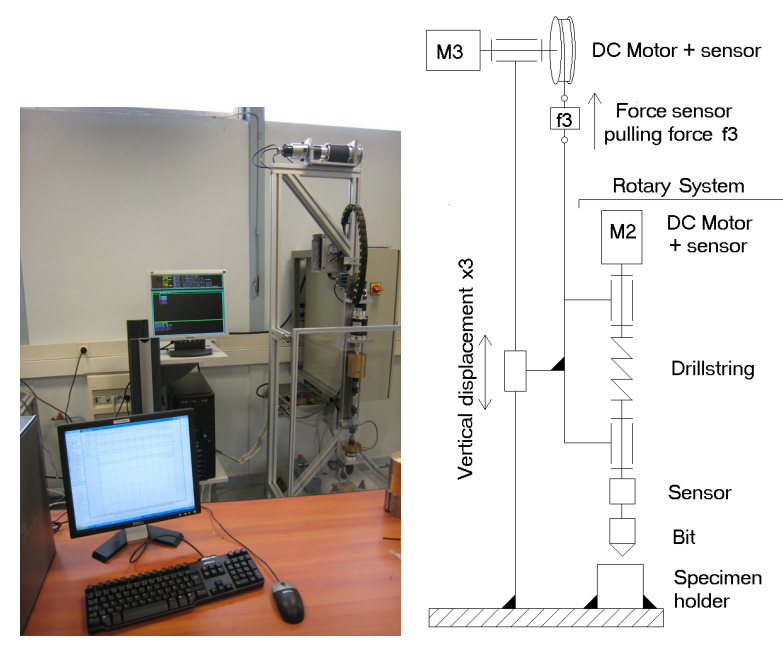

Fig. 1. Experimental setup and the schematic of the rotary system

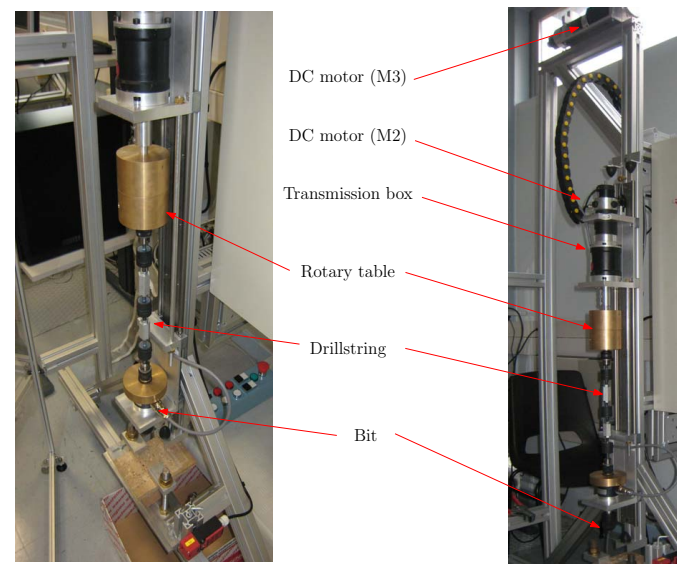

Fig. 2. Photos of the rotary system

- DC motor (M2) provides necessary mechanical energy to make the system rotate. A rotary speed sensor is built.

- Transmission box connects the motor and the rotary table.

- Rotary table is a large disc-shaped inertia coupled to the drill-string which drives the rotating motion.

- Drillstring is a sequence of tubes that connect the rotary table and the bit. It has a low rotational stiffness. 
- Quadrature encoder. measures the rotary angle of the bit. This rotary angle is then used to compute the rotary speed at the bit.

- Bit is the cutting tool used to drill.

SUPPORT PLATFORM platform consists of:

- DC motor (M3) is employed to move the rotary system vertically and vary the weight applied to the bit during the drilling process (WoB). A rotary speed sensor and a quadrature encoder are built in.

- Specimen holder fixes the specimen below the drill bit.

- Support structure is used to support other physical parts and provide necessary safety mechanism.

- Tension force sensor is used to measure the vertical force applied on the rotary system.

POWER SYSTEM: power supply and peripheral circuits. It provides electrical energy for other parts of the experiment setup.

COMPUTER BASED CONTROL SYSTEM: Host PC and xPC Target

- Host PC It is used to create applications using Matlab, Simulink. It serves as an interface to command the target PC.

- Target PC It is xPC Target real-time platform used for controlling the whole setup . The target PC downloads executable code created using Matlab, Simulink and Stateflow software from the host PC and interacts with the host PC and hardware system to perform tasks.

\section{A. Software}

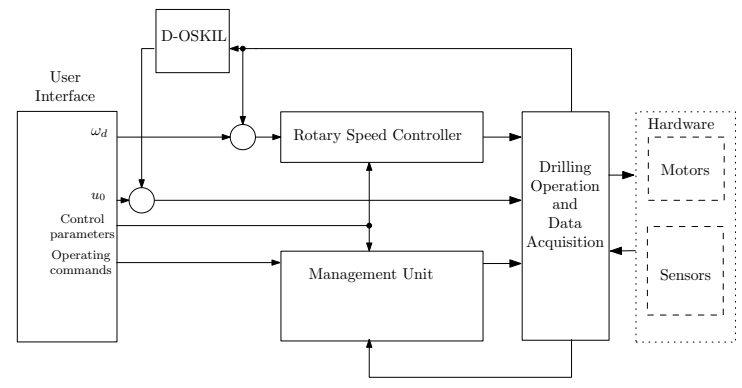

Fig. 3. Function blocks of the platform

Basic software platform was constructed using Matlab, Simulink and Stateflow software on the host PC. Function blocks of the program are depicted in Fig.3

\section{B. Drilling Tools and Drilled Specimen}

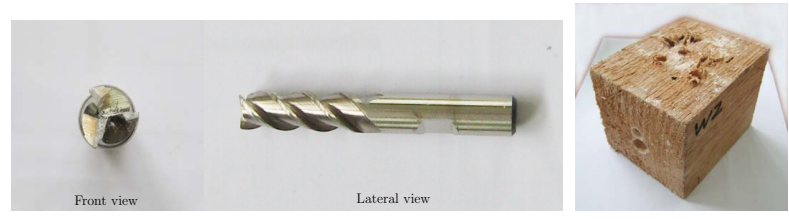

Fig. 4. The drill bit (left), and the cedar wood block specimen (right)
Suitable combinations of drill bit and specimen were selected to meet the criterion that the oscillation must be able to be removed when the applied WoB is small. An oil drilling system in the field usually has a circulation system to lubricate and refrigerate the contact between the rock and the bit.As we do not have such facility in the setup, the swarf removal ability of the bit is vital. If the swarf is not removed as fast as it is produced, the flutes will clog and prevent the tool drilling efficiently, causing vibration, tool wear and overheating. Thus we chose the milling cutter shown in Fig.4 as the drill bit which satisfies our criterion. Cedar wood blocks (Fig.4) were used as the specimens in our test.

\section{Drillstring Characteristic Measurement}

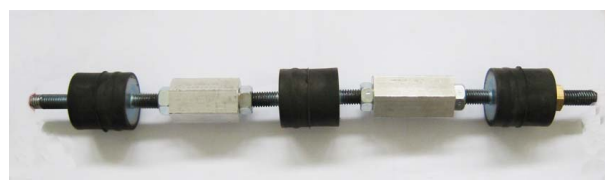

Fig. 5. The drillstring

As depicted in Fig.5, the drillstring was used in this experimental setup. An experiment was carried out to determine the rotational stiffness of the drillstring. A rotational torque $(\tau)$ in the step of 0.1 from 0 to $3 \mathrm{~N} \cdot \mathrm{M}$ was applied to the rotary table with the bit fixed. Corresponding rotational angles $(\theta)$ of the rotary table was plotted with respect to the torque. Fig. 6 shows the relation is approximately linear within the range. Then rotational stiffness was determined through linear regression.

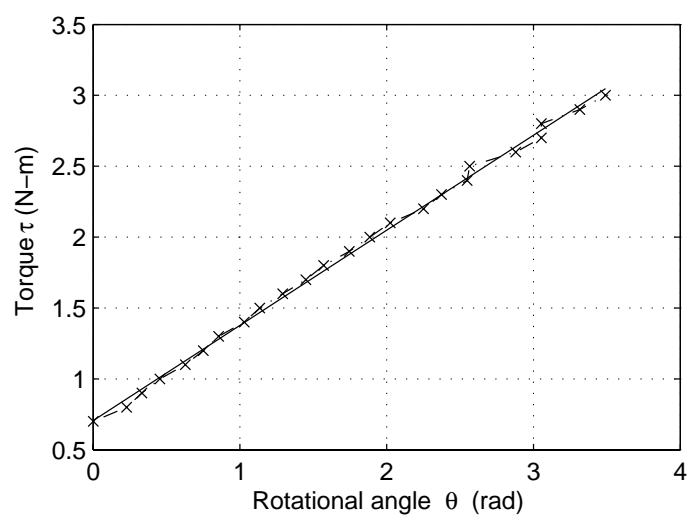

Fig. 6. The torque vs. rotational angle characteristics of the drillstring

$$
\tau=\tau_{0}+k \cdot \theta
$$

$k=0.6706 \mathrm{~N} \cdot \mathrm{m} / \mathrm{rad} \tau_{0}=0.7069 \mathrm{~N} \cdot \mathrm{m}$, where $k$ is the rotational stiffness of the drillstring and $\tau_{0}$ is the static friction torque without causing rotation. 


\section{MODEL AND PARAMETER IDENTIFICATION}

\section{A. Drillsting Model}

Various kind of models have been used in literature to describe drillstring systems (for example [2] and [3]). The lumped model used in the study of the D-OSKIL mechanism is a two-degree-of-freedom model with two inertial masses $J_{r}$ and $J_{b}$, locally damped by $d_{r}$ and $d_{b}$. The inertias are coupled with each other by an elastic shaft of stiffness $k$ and damping $c$. The variables $\varphi_{r}$ and $\varphi_{b}$ stand for the rotary and the bit angle. The rotary torque control signal $\mathrm{v}$ is used to regulate the rotary angular velocity $\dot{\varphi}_{r}$. The $T_{o B}$ (Torque on $\mathrm{Bit}$ ) represents the total friction torque over the drill bit. The model equations are as follows:

$$
\begin{gathered}
J_{r} \ddot{\varphi}_{r}+c\left(\dot{\varphi}_{r}-\dot{\varphi}_{b}\right)+k\left(\varphi_{r}-\varphi_{b}\right)+d_{r} \dot{\varphi}_{r}=v \\
J_{b} \ddot{\varphi}_{b}+c\left(\dot{\varphi}_{b}-\dot{\varphi}_{r}\right)+k\left(\varphi_{b}-\varphi_{r}\right)+d_{b} \dot{\varphi}_{b}=-T_{o B}
\end{gathered}
$$

In constants above, the sub-script ' $r$ ' and 'b' stands for rotary table and bit, respectively.

The $T_{o B}$ is given by the product of $\mu\left(\dot{\varphi}_{b}\right)$, which describes the normalized (dimensionless) torsional bit-rock friction (different bit-rock friction models are presented in [4]), and the normal force $u(\mathrm{WoB})$, i.e.

$$
T_{o B}=\mu\left(\dot{\varphi}_{b}\right) \cdot u, \quad u=u_{0}+\tilde{u}
$$

The effective value of $T_{o B}$ can be modified by controlling the tension force, $\tilde{u}$, of the drillstring. We assume that a counterweight force $\tilde{u}, \tilde{u} \in\left[-u_{0}, 0\right]$, can be applied to counteract the gravitational nominal force $u_{0}=M g(M$ is the total drillstring mass and $g$ is the gravity).

This lumped model has the following state-space representation:

$$
\dot{x}=A x+B \nu+H \mu\left(x_{3}\right) u
$$

with

$$
\begin{gathered}
A=\left(\begin{array}{ccc}
0 & 1 & -1 \\
-\frac{k}{J_{r}} & -\frac{d_{r}+c}{J_{r}} & \frac{c}{J_{r}} \\
\frac{k}{J_{b}} & \frac{c}{J_{b}} & -\frac{c+d_{b}}{J_{b}}
\end{array}\right) \\
B=\left(\begin{array}{c}
0 \\
\frac{1}{J_{r}} \\
0
\end{array}\right), H=\left(\begin{array}{c}
0 \\
0 \\
-\frac{1}{J_{b}}
\end{array}\right)
\end{gathered}
$$

where the state $x=\left[x_{1}, x_{2}, x_{3}\right]^{T}$ is defined as follows:

$$
\begin{aligned}
& x_{1}=\varphi_{r}-\varphi_{b} \\
& x_{2}=\dot{\varphi}_{r} \\
& x_{3}=\dot{\varphi}_{b}
\end{aligned}
$$

Realistic values for the parameters which were identified from our experimental setup are given in Table .

\section{B. Parameter Identification}

In the real drilling system in the field, there exists significant friction between drillstring and the surrounding, e.g., borehole wall and the drilling mud, which is reflected by the damping coefficient $c$ in the model shown in Section III-A. In our test, as the drillstring is directly exposed to the air, such friction is negligible, i.e. $c=0$.

Except the stiffness $k$ which has been calculated in Section II-C, the other four parameters of the system model were identified as follows.

Firstly, the drillstring linked between the rotary table and the bit was removed to identify $J_{r}$ and $d_{r}$. Then step torque produced by the DC motor (M2) was applied on the rotary table periodically, and the rotary speed $\left(\dot{\varphi}_{r}\right)$ response of the rotary table was recorded. The step value of the applied torque was determined to keep the rotary table speed close to the speed used in the drilling $\left(\dot{\varphi}_{r} \approx w_{d}=5 \mathrm{rad} / \mathrm{s}\right)$. The system can be represented by Equation (3).

$$
J_{r} \ddot{\varphi}_{r}+d_{r} \dot{\varphi}_{r}=\tau_{r}
$$

Secondly, for $J_{b}$ and $d_{b}$, we used such a steel pole of high stiffness as the drillstring that the rotation difference between the rotary table and the bit is zero, i.e. $\dot{\varphi}_{r}-\dot{\varphi}_{b}=0$. The system can be represented by Equation (4). Then we obtained the step response of the rotary speed $\left(\dot{\varphi}_{r+b}\right)$.

$$
\left(J_{r}+J_{b}\right) \ddot{\varphi}_{r+b}+\left(d_{r}+d_{b}\right) \dot{\varphi}_{r+b}=\tau_{r+b}
$$

By processing the step response results, we can calculate the parameters of the first order model as follows.

In s-domain:

$$
\begin{aligned}
& \frac{\dot{\varphi}_{r}(s)}{\tau_{r}(s)}=\frac{1}{J_{r} s+d_{r}}=\frac{24.60}{s+6.172} \\
& \frac{\dot{\varphi}_{r+b}(s)}{\tau_{r+b}(s)}=\frac{1}{\left(J_{r}+J_{b}\right) s+\left(d_{r}+d_{b}\right)}=\frac{21.81}{s+5.63}
\end{aligned}
$$

Giving:

$$
\begin{aligned}
& J_{r}=0.04065 \mathrm{Kg} \cdot \mathrm{m}, \quad d_{r}=0.25088 \mathrm{~s}^{-1} \\
& J_{b}=0.0052 \mathrm{Kg} \cdot \mathrm{m}, \quad d_{b}=0.00726 \mathrm{~s}^{-1}
\end{aligned}
$$

Steps for experimental validations, not reported here for lack of space, were performed on the setup.

\section{CONTROL ARCHITECTURE}

\section{A. Rotary table speed control loop}

A speed control loop is designed to regulate the output rotary table speed $\dot{\varphi}_{r}$ to a desired value $\omega_{d}$ (typical value for $\omega_{d}$ is $5 \mathrm{rad} / \mathrm{s}$ ).

Though many advanced speed control law, (i.e. $H_{\infty}$ ), are available in the literature, oil well drilling often operates with reduced-order simple control laws. We adopted the control structure proposed in [1]:

$$
v=\left[k_{1}+\frac{k_{2}}{s}\right]\left(\omega_{d}-\dot{\varphi}_{r}\right)-k_{3}\left(\dot{\varphi}_{r}-\dot{\varphi}_{b}\right)
$$


which can also be written as:

$$
v=k_{1}\left(\omega_{d}-x_{2}\right)+k_{2} x_{4}-k_{3}\left(x_{2}-x_{3}\right), \quad \dot{x}_{4}=\omega_{d}-x_{2}
$$

then the closed-loop equation take the form,

$$
\dot{x}=A_{c l} x+B_{c l} \omega_{d}+H_{c l} \mu(x) u
$$

with the state of dimension four, i.e. $x=\left[x_{1}, x_{2}, x_{3}, x_{4}\right]$, and with the $A_{c l}, B_{c l}, H_{c l}$ given as follows:

$$
\begin{gathered}
A_{c l}=\left(\begin{array}{cccc}
0 & 1 & -1 & 0 \\
-\frac{k}{J_{r}} & -\frac{d_{r}+c+k_{1}+k_{3}}{J_{r}} & \frac{c+k_{3}}{J_{r}} & \frac{k_{2}}{J_{r}} \\
\frac{k}{J_{b}} & \frac{c}{J_{b}} & -\frac{c+d_{b}}{J_{b}} & 0 \\
0 & -1 & 0 & 0
\end{array}\right) \\
B_{c l}=\left(\begin{array}{c}
0 \\
\frac{k_{1}}{J_{r}} \\
0 \\
1
\end{array}\right), H_{c l}=\left(\begin{array}{c}
0 \\
0 \\
-\frac{1}{J_{b}} \\
0
\end{array}\right)
\end{gathered}
$$

The gains values can be designed using pole placement for the closed loop system. The details about the speed controller gain tuning in our case is presented in later section.

\section{B. Oscillation characteristics}

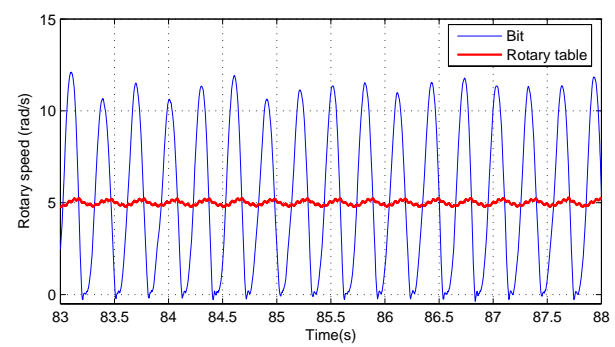

Fig. 7. Stick-slip measured in our experimental drilling system

The oscillation pattern shown by Fig. 7 is generated with our testbed setup. They reflect the main characteristic of the drill oscillations: first oscillations will present a bias term, and second they are dominated by a main harmonic with a relatively well-defined period. Thus, the SBDF (Sinusoid plus bias describing function) method can be used as a first approximation to predict possible limit cycle and to study its stability. The detail analysis given in [1] demonstrates the relationships among system bandwidth $\left(\omega_{n}\right)$, WoB, and the possibility to enter into oscillation. On the basis of this analysis, oscillation may be eliminated either by changing $\omega_{n}$, and/or by reducing the $\mathrm{WoB}$ magnitude.

Keeping WoB low will be a too conservative strategy. High-performance drilling operation usually takes place in a region in the plane $\left(\mathrm{WoB}-\omega_{n}\right)$, where potential oscillations may occur. Typical values of $\omega_{n}$ are in the range $[20,30] \mathrm{rad} / \mathrm{s}$. So in the design of the control law we account for this particularity assuming that the nominal system operation parameters are taken within this zone.

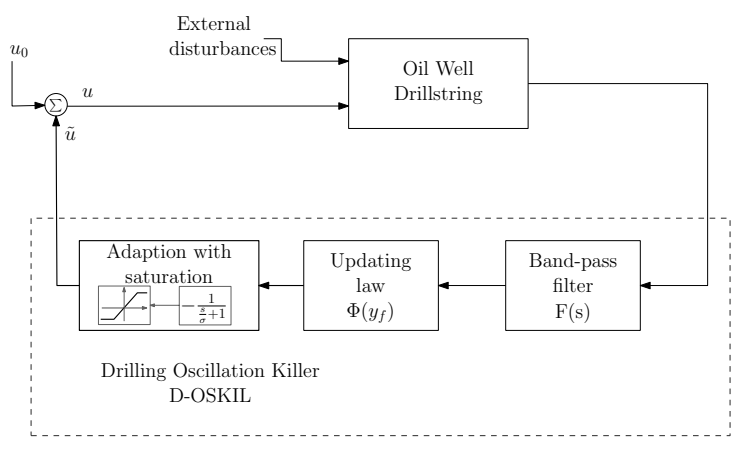

Fig. 8. Block scheme of the D-OSKIL mechanism

\section{D-OSKIL Mechanism}

The D-OSKIL mechanism to be implemented is thus built under the following base-lines:

- nominal values for $\left(\mathrm{WoB}-\omega_{n}\right)$ are in the potential stable limit cycle zone but the system trajectories are at their equilibrium, i.e. $u=u_{0}$ and $\dot{\varphi}_{b}=\omega_{d}$. Then, stick-slip limit cycles are possible under a substantial perturbation. As it is often the case during the real drill operation, variation on the rock friction characteristics may bring the closed-loop system trajectories outside the local stable zone producing stick-slip cycles.

[1]

- the oscillation is suppressed by reducing the WoB,i.e. adjusting the control signa $\tilde{u}$, so that the local attraction domain is enlarged about the equilibrium points until system trajectories are attracted to them.

- after that, the WoB will have to be recovered to its nominal value to continue with the proper drilling task, i.e. $u \rightarrow u_{0}$. This is possible as long as the system trajectories are kept within the local attraction domain, by a slow variation of the WoB, see [1].

One possible variation for the D-OSKIL mechanism is the following one (the superscript ${ }^{*}$ indicates steady-state values):

$$
\begin{aligned}
y & =C e=\dot{\varphi}_{b}-\omega_{d}, \\
y_{f} & =F(s) y \\
\frac{1}{\sigma} \dot{\tilde{u}} & =-\tilde{u}-\operatorname{sat} t_{-u_{0}}^{0}\left\{\Phi\left(y_{f}\right)\right\} \\
\Phi\left(y_{f}\right) & =\lambda \cdot y \cdot \operatorname{sgn}\left(\mu\left(y_{f}\right)\right), \quad \lambda \geq 0 \\
u & =u_{0}+\tilde{u}
\end{aligned}
$$

where $e=x-x^{*}, \tilde{u}(0)=0, \sigma>0$ and the assumption of the possibility to measure or estimate $\dot{\varphi}_{b}$ is made.

\section{Remarks}

Equation (7) selects a suitable output reflecting the stickslip oscillations. This choice is not unique, and it may depend on the signal-to-noise ratio of the considered output. To simplify matters, the selected output is $y=\dot{\varphi}_{b}-\omega_{d}$.

Equation (8) defines a band-pass filter F(s) used to extract the main oscillatory component. A filter of the following 
form can be used.

$$
F(s)=\frac{K_{f} s}{\left(s+\omega_{1}\right)\left(s+\omega_{2}\right)}
$$

with $\omega_{1}=\omega_{0}-\Delta \omega>0$, and $\omega_{2}=\omega_{0}+\Delta \omega>0\left(\omega_{0}\right.$ is the frequency of the main oscillation component). The value of $K_{f}$ and $\Delta \omega$ are designed such that $\left|F\left(j \omega_{0}\right)\right| \approx 1$, and frequencies away from $\omega_{0}$ are filtered out. $\omega_{0}$ can be obtained from a frequency estimator. In this test run, and for simplicity it was approximately identified from the experimental data.

Equation (9) describes the control law, with output saturation. It forces $\tilde{u} \in\left(-u_{0}, 0\right], \forall t \geq 0$. Thus, in Equation (11) $u$ is bounded in $\left(0, u_{0}\right] \cdot \frac{1}{\sigma}$ can be understood as a time constant of the controller.

In Equation (10) $\Phi\left(y_{f}\right)$ is a nonlinear function which must be designed to ensure system stability, i.e. As $\dot{\varphi}_{b} \rightarrow$ $\omega_{d}, \tilde{u} \rightarrow 0$. The paper [1] shows relevant stability analysis and presents the criteria to select proper values of $\sigma$ and $\lambda$. Details about tuning of the two DOSKIL control parameters $\sigma$ and $\lambda$ on the experimental setup will be demonstrated in later section.

\section{EXPERIMENTAL VALIDATION}

\section{A. Weight on Bit force Regulation}

A PI controller with following structure is used to regulate the $\mathrm{WoB}$ during the drilling process.

$$
C_{3}=c_{0}-\left(W o B_{d}-W o B\right)\left(\frac{K_{i 3}}{s}+1\right) K_{p 3}
$$

$C_{3}$ is the output torque of the controller (input of the DC motor M3) and $c_{0}$ is the offset torque corresponding to the total weight of the rotary system. $W o B$ and $W o B_{d}$ are respectively weight on bit measured and desired.

The ability of the PI controller directly affects the performance of the D-OSKIL mechanism. For instances, larger overshoot may significantly disturb the torque on the bit and potentially cause some oscillations. The performance of the closed-loop controller which operated while drilling was checked. The step responses obtained with our settings, $K_{i 3}=5.9504$, and $K_{p 3}=2.3$, shows that the rise time is less than 0.2 second, overshoot is less than $35 \%$, and steadystate error is less than $0.5 \%$. The performance is satisfactory in our test.

\section{B. D-OSKIL}

The D-OSKIL mechanism shown by the equation 9-13 contains two main control parameters $\sigma$ and $\lambda$. The other two parameters $\left(\omega_{0}\right.$ and $\Delta \omega$ ) for the bandpass filter were determined by identifying the oscillation frequency in the experimental result. In our case, the main oscillation frequency is around $3.2 \mathrm{~Hz}$. Thus we chose $\omega_{0}=20 \mathrm{rad} / \mathrm{s}$ and $\Delta \omega=$ 0.5 .

$\frac{1}{\sigma}$ can be understood as a time constant which controls the speed of transition from oscillation regime to stabilization period and the stabilization time for the Wob, i.e. how fast the WoB recovers to its nominal value. Together with $\sigma, \lambda$ determines the magnitude of the output control signal $\tilde{u}$. It have to be tuned properly to make the output signal reflect the magnitude of the oscillation. The effects of these two parameters on the control performance are discussed in [1].

It must be noted that the remaining oscillations (e.g. high frequency torsional vibrations) always exists and our goal is to eliminate the stick-slip oscillations. From a practical point of view, above factors must be balanced and the parameters values with $\sigma=0.15$ and $\lambda=5$ has been proved to be valid in our experiment. The result are shown in Fig.10.

\section{Experimental results}
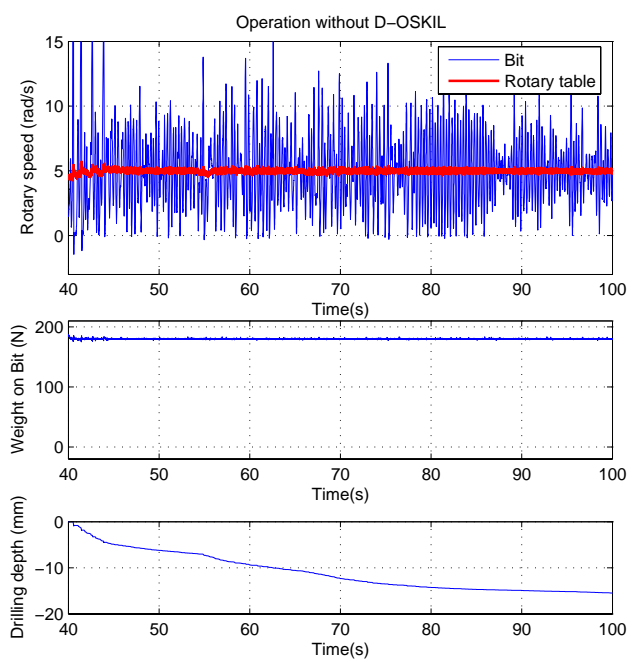

Fig. 9. Experiment result without D-OSKIL scheme: Overall view (Upper) and detailed (Bottom) view
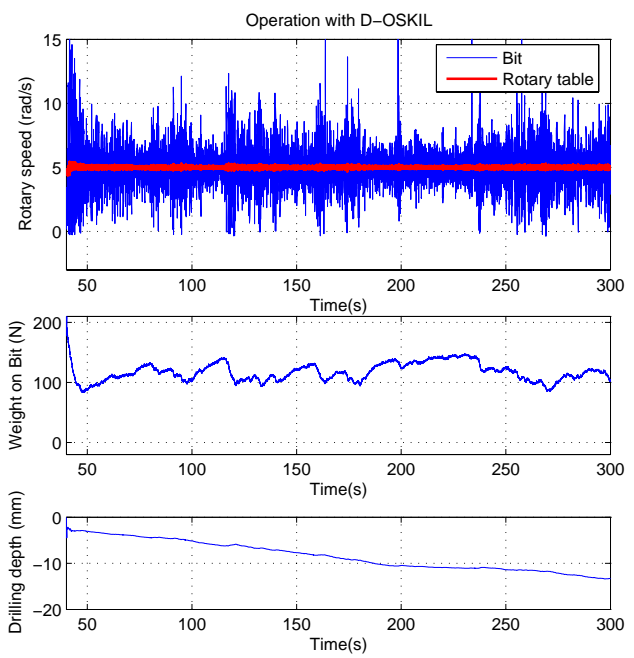

Fig. 10. Experiment result with D-OSKIL scheme: Overall view (Upper) and detailed (Bottom) view scheme

Results of the experiments performed with the nominal weight $u_{0}=180 \mathrm{~N}$ respectively with and without D-OSKIL activated are shown in Fig.9 and Fig.10. Each figure gives an overall view of the plot for each test result. Since the drilling 


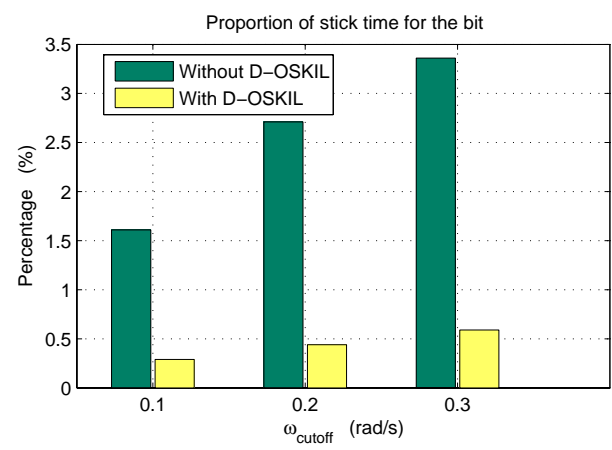

Fig. 11. Proportion of stick time for the bit in the whole drilling process

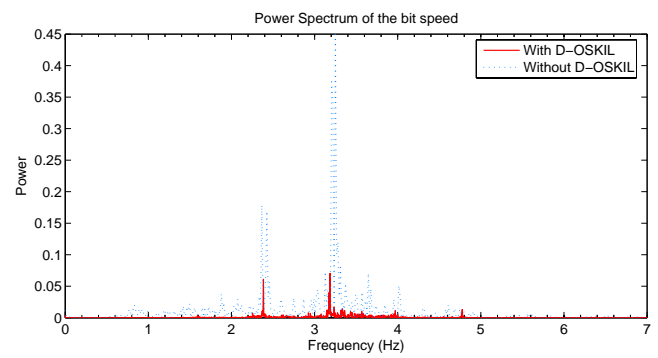

Fig. 12. Comparison between power spectrums of the bit speed with and without D-OSKIL scheme

positions on the wood specimens were different for each test and the wood is heterogeneous mixture, we have to assume that the condition is approximately same for each test.

In these figures, the typical profiles of both the bit and the rotary table rotary speed, system Wob, and drilling depth $\left(x_{3}\right)$ are shown. As it can be observed in Fig. 9, without D-OSKIL mechanism, the system is under a stickslip oscillation regime. The sustained stick-slip phenomenon is significant from 78 seconds to 84 seconds.

In Fig.10, the D-OSKIL mechanism is able to suppress the stick-slip oscillation. The WoB recovers after the stick-slip oscillation has been eliminated. The remaining oscillations always exist due to the nature of the closed-loop system, and some other factors (e.g. vertical vibrations). Compared with Fig.9, the penetration rate (gradient of the plot of $x_{3}$ ) of the drilling with D-OSKIL mechanism is smaller than that without D-OSKIL mechanism.

In addition to the simple visual evaluation of the OSKIL mechanism, we used some analytical methods to analyze the experiment data. Following analysis results are obtained by processing the experiment data from 40s to 100s in Fig.9 and the data from 40 s to 300 s in Fig.10. One major feature of harmful stick-slip phenomenons is that the bit stops rotating regularly. We propose to use the proportion of stick time for the bit in the whole drilling process as an evaluation factor. Due to the inaccuracy in the measurement, the moment when the rotary speed at the bit is below a certain small value $\left(\omega_{\text {cutoff }}\right)$ is considered as bit sticking.

Another analysis is performed in frequency domain. We got the power spectrum of the bit speed using Fast Fourier
Transform (FFT). For clearer comparison between these two, the two spectrums are plotted in Fig.12. It is observed that the stick-slip components with frequency around $3.3 \mathrm{~Hz}$ have been suppressed to 12 percent of the original value, because the bandpass frequency of the filter in D-OSKIL mechanism was set to around $20 \mathrm{rad} / \mathrm{s}$. It is obvious that the stick-slip oscillation can be suppressed effectively.

\section{CONCLUSION}

This paper presents the implementation of the D-OSKIL mechanism proposed in [1] to suppress stick-slip oscillations in a oil well drilling system. We have constructed a test platform to emulate the oil well drillstring system in the field, and implemented the D-OSKIL mechanism on the setup.

Several tests have been conducted to investigate the performance of the D-OSKIL mechanism in our circumstance. The test scenario and results have been illustrated in this paper. The results show that this mechanism is able to effectively suppress stick-slip oscillations occurred during drilling process.

\section{APPENDIX}

TABLE I

TABLE OF PARAMETER VALUES USED IN THE TEST

\begin{tabular}{|c|r|c|}
\hline$J_{r}$ & 0.04065 & {$\left[\mathrm{Kg} \cdot \mathrm{m}^{2}\right]$} \\
$J_{b}$ & 0.0052 & $\mathrm{Kg} \cdot \mathrm{m}^{2}$ \\
$k$ & 0.67 & {$[\mathrm{Nm} / \mathrm{rad}]$} \\
$c$ & 0 & {$\left[1 / \mathrm{s}^{2}\right]$} \\
$d_{r}$ & 0.25088 & {$[1 / \mathrm{s}]$} \\
$d_{b}$ & 0.00726 & {$[1 / \mathrm{s}]$} \\
$\mu_{c}$ & 0.015 & {$[-]$} \\
$\mu_{s}$ & 0.025 & {$[-]$} \\
$\nu_{s}$ & 0.01 & {$[\mathrm{rad} / \mathrm{s}]$} \\
$\sigma_{0}$ & 25 & {$[\mathrm{rad} / \mathrm{s}]$} \\
$\sigma_{1}$ & 0.05 & {$[\mathrm{rad} / \mathrm{s}]$} \\
$u_{0}$ & 180 & $\mathrm{Kg} \cdot \mathrm{m} / \mathrm{s}^{2}$ \\
$\omega_{d}$ & 5 & $\mathrm{rad} / \mathrm{s}$ \\
$k_{1}$ & 2 & {$[\mathrm{Nm} / \mathrm{rad} / \mathrm{s}]$} \\
$k_{2}$ & 1.8 & {$[\mathrm{Nm} / \mathrm{rad}]$} \\
$k_{3}$ & 0.034 & {$[\mathrm{Nm} / \mathrm{rad} / \mathrm{s}]$} \\
$\omega_{0}$ & 20 & $\mathrm{rad} / \mathrm{s}$ \\
$\Delta_{\omega}$ & 0.5 & $\mathrm{rad} / \mathrm{s}$ \\
\hline
\end{tabular}

\section{REFERENCES}

[1] C. Canudas-de Wit, F. Rubio, and M. Corchero, "D-oskil: A new mechanism for controlling stick-slip oscillations in oil well drillstrings," Control Systems Technology, IEEE Transactions on, vol. 16, no. 6, pp. 1177-1191, Nov. 2008.

[2] R. Leine, D. Van Campen, and W. Keultjes, "Stick-slip whirl interaction in drillstring dynamics," Journal of Vibration and Acoustics, vol. 124, pp. 209-220, 2002.

[3] P. Spanos, A. Chevallier, N. Politis, and M. Payne, "Oil well drilling: A vibrations perspective," The Shock and Vibration Digest, vol. 35, no. 2, pp. 81-99, 2003.

[4] E. Navarro-Lopez and R. Suarez-Cortez, "Modelling and analysis of stick-slip behaviour in a drillstring under dry friction," in Congress of the Mexican Association of Automatic Control, 2004, pp. 330-335. 